\title{
Photonic generation of high-frequency microwave signals utilizing a multi-transverse-mode vertical-cavity surface-emitting laser subject to two-frequency orthogonal optical injection
}

\author{
Ana Quirce, ${ }^{1,2}$ Angel Valle, ${ }^{1, *}$ Hong Lin, ${ }^{3}$ David W. Pierce, ${ }^{3}$ and Yu Zhang ${ }^{3}$ \\ ${ }^{1}$ Instituto de Física de Cantabria, CSIC-Universidad de Cantabria, Avda. Los Castros s/n, Santander E-39005, Spain \\ ${ }^{2}$ Departamento de Física Moderna, Universidad de Cantabria, Avda. Los Castros s/n, Santander E-39005, Spain \\ ${ }^{3}$ Department of Physics \& Astronomy, Bates College, Lewiston, Maine 04240, USA \\ ${ }^{*}$ Correspoinding author: valle@ifca.unican.es
}

Received June 12, 2012; revised October 5, 2012; accepted October 9, 2012; posted October 11, 2012 (Doc. ID 170436); published November 8, 2012

\begin{abstract}
We study photonic microwave signal generation obtained when single- and multi-transverse-mode vertical-cavity surface-emitting lasers (VCSELs) are subject to two-frequency orthogonal optical injection. Our calculations show that broadly tunable microwave signals can be obtained in these systems. The response of the multi-transversemode VCSEL is enhanced with respect to that obtained with a similar single-transverse-mode VCSEL subject to the same two-frequency orthogonal optical injection. The extra degree of freedom given by the multi-transverse-mode operation of the VCSEL under two-frequency orthogonal optical injection enhances the performance of the photonic microwave generation system, because the higher-order transverse mode is excited with a much larger amplitude than that of the fundamental transverse mode. Periodic oscillations are obtained for a very wide range of frequency detunings between the optical injections and transverse modes. A relative maximum of the microwave signal amplitude is obtained when the frequency of one of the optical injections is very close to the frequency of the orthogonally polarized fundamental mode of the VCSEL. Periodic oscillations are demonstrated for symmetric and asymmetric values of the injection strengths. Wide tuning ranges, extended into the $\mathrm{THz}$ band, are obtained in our system. Our results show that the proposed microwave signal generation mechanism is independent of the polarization of the master lasers. (ㄱ) 2012 Optical Society of America
\end{abstract}

OCIS codes: $\quad 140.5960,250.7260,140.3520$.

\section{INTRODUCTION}

Optical injection in semiconductor lasers, and particularly in vertical-cavity surface-emitting lasers (VCSELs), has been studied extensively in recent years [1-15]. VCSELs are attractive light sources because of their compactness, low power consumption, circular output beam, dense packaging, singlelongitudinal-mode operation, fast response, and low cost [2]. Emission in multiple transverse modes is usually found in VCSELs [16] as a result of the spatial-hole burning effect [17]. Furthermore, due to the surface emission and cylindrical symmetry, VCSELs lack strong polarization anisotropy and may undergo polarization switching (PS) [18]. The lack of polarization anisotropies and the multi-transverse-mode behavior of VCSELs provide new features to the rich nonlinear dynamics induced by optical injection. Locking of the frequency of the injected (slave) VCSEL to that of the injecting (master) laser enhances the VCSEL modulation bandwidth $[3,4]$. Orthogonal optical injection in VCSELs, in which the polarization of the injected signal is perpendicular to the linear polarization of the VCSEL, has received a lot of attention [6-15]. It has been shown that the VCSEL switches its polarization to that of the master laser when increasing the injection strength [6]. Most of these studies have been performed for single-transverse-mode VCSELs [6-12]. The effect of orthogonal optical injection on multi-transverse-mode VCSELs has been also recently studied, showing that PS can be induced in all the transverse modes [13-15]. Recent experimental work has demonstrated single-mode [19] and multimode [20] VCSEL-by-VCSEL optical injection locking for obtaining integrated low-cost, high-speed communication modules [19].

Microwave signals can be generated by using nonlinear dynamics of optically injected semiconductor lasers [21-30]. Photonic microwave generation finds applications in radioover-fiber technology that holds great promise for $4 \mathrm{G}$ mobile communications systems [23]. Both single-frequency [21-26] and two-frequency optically injected semiconductor lasers [27-30] have been considered. Tunable narrow-linewidth microwave signals have been generated by using the period-one oscillations that appear when a semiconductor laser is subject to single-frequency optical injection [23]. These oscillations have reached frequencies beyond $100 \mathrm{GHz}$ [25] with tuning ranges limited to several tens of gigahertz [28]. Recently, a dual-beam optically injected single-mode distributed feedback (DFB) laser has been used to generate a very-high-frequency (121.7 GHz) microwave signal [28]. Theoretical results show that a wide continuous tuning range of more than $100 \mathrm{GHz}$ can be obtained by adjusting the detuning frequency of the two 
master lasers [29]. However, only experimental tuning ranges around $20 \mathrm{GHz}$, limited by the bandwidth of the photodetector, have been demonstrated [28]. Very recently, a theoretical study of a multi-transverse-mode VCSEL subject to twofrequency parallel optical injection has shown that broadly tunable microwave signals can be generated [30]. In the parallel optical injection, the polarization of the injected signal is parallel to the linear polarization of the VCSEL. The response of multi-transverse-mode VCSELs under two-frequency parallel optical injection is larger than that obtained with similar single-transverse-mode VCSELs subject to the same optical injection [30]. In this way the maximum frequency of the generated microwave signals can be substantially increased if multimode VCSELs are used instead of single-mode VCSELs [30].

In this work we extend the previous study to consider twofrequency orthogonal optical injection. The aim of our work is to show that the method of generation of microwave signals proposed in [30] is also valid when the polarization of the two-frequency optical injection is orthogonal to the linear polarization of the VCSEL. In this way the microwave signal generation can be obtained independently of the polarization of the master lasers. The dynamics of the two linear polarizations of each of the transverse modes of the VCSEL is found using the model of [30], modified to consider orthogonal optical injection. We analyze the nonlinear dynamics of the system, making special emphasis on the periodic oscillations observed at large injection strengths, useful for photonic microwave signal generation. Simulation of single-transversemode VCSELs with the extra degree of freedom given by the polarization of the device show that periodic oscillations can frequency of the orthogonal polarization of the fundamental mode of the VCSEL. Wide tuning ranges, extending into the terahertz band, are demonstrated in our system.

Our paper is organized as follows. In Section 2 the theoretical model is presented. Section 3 is devoted to the singletransverse-mode VCSEL subject to two-frequency orthogonal optical injection. In Section 4 we study the multi-transversemode VCSEL subject to the same type of injection. In Section 5 we provide a discussion of our results. Finally, Section 6 is devoted to the summary and conclusions of the paper.

\section{THEORETICAL MODEL}

Our model is based on a spatially dependent dynamical model of a multi-transverse-mode VCSEL subject to single-frequency orthogonal optical injection [13]. In this paper we extend it to consider a two-frequency optical injection, or equivalently, we modify the model of [30] in order to consider orthogonal optical injection. The simulated cylindrically symmetric weakly index-guided structure, together with the complete details of the model, can be found in [13]. Subscripts $x$ and $y$ will be used to denote the orthogonal polarization directions. We will consider a small value of the index step of the waveguide (0.09) in such a way that the appropriate transverse modes of the structure are the $\mathrm{LP}_{m n}$ modes. Here we treat the case of VCSELs that can operate in the fundamental $\left(\mathrm{LP}_{01}\right)$ and in the first-order $\left(\mathrm{LP}_{11}\right)$ transverse modes. Subscripts 0,1 will be used to denote the $\mathrm{LP}_{01}$ and $\mathrm{LP}_{11}$ modes, respectively. The equations describing the polarization and transverse mode behavior of the VCSEL with two-frequency orthogonal optical injection read $[\underline{13}, \underline{30}]$

$$
\begin{aligned}
& \dot{E}_{0 x}=k(1+i \alpha)\left(E_{0 x}\left(g_{0 x}-1\right)+i E_{0 y} g_{0 x y}\right)-\left(\gamma_{a}+i \gamma_{p 0}\right) E_{0 x}+\sqrt{\frac{\beta}{2}}\left(\sqrt{\bar{N}+\bar{n}} \xi_{0+}(t)+\sqrt{\bar{N}-\bar{n}} \xi_{0-}(t)\right), \\
& \dot{E}_{0 y}=k(1+i \alpha)\left(E_{0 y}\left(g_{0 y}-1\right)-i E_{0 x} g_{0 y x}\right)+\left(\gamma_{a}+i \gamma_{p 0}\right) E_{0 y}+\frac{\kappa_{01}}{\tau_{i n}} e^{i \Delta \omega_{1} t}+\frac{\kappa_{02}}{\tau_{i n}} e^{i \Delta \omega_{2} t}-i \sqrt{\frac{\beta}{2}}\left(\sqrt{\bar{N}+\bar{n}} \xi_{0+}(t)-\sqrt{\bar{N}-\bar{n}} \xi_{0-}(t)\right), \\
& \dot{E}_{1 x}=k(1+i \alpha)\left(E_{1 x}\left(g_{1 x}-\kappa_{r}\right)+i E_{1 y} g_{1 x y}\right)+i \gamma_{p}^{t r} E_{1 x}-\left(\gamma_{a}+i \gamma_{p 1}\right) E_{1 x}+\sqrt{\frac{\beta}{2}}\left(\sqrt{\bar{N}+\bar{n}} \xi_{1+}(t)+\sqrt{\bar{N}-\bar{n}} \xi_{1-}(t)\right) \text {, } \\
& \dot{E}_{1 y}=k(1+i \alpha)\left(E_{1 y}\left(g_{1 y}-\kappa_{r}\right)-i E_{1 x} g_{1 y x}\right)+i \gamma_{p}^{t r} E_{1 y}+\left(\gamma_{a}+i \gamma_{p 1}\right) E_{1 y}+\frac{\kappa_{11}}{\tau_{i n}} e^{i \Delta \omega_{1} t}+\frac{\kappa_{12}}{\tau_{i n}} e^{i \Delta \omega_{2} t}-i \sqrt{\frac{\beta}{2}}\left(\sqrt{\bar{N}+\bar{n}} \xi_{1+}(t)-\sqrt{\bar{N}-\bar{n}} \xi_{1-}(t)\right) \text {, } \\
& \frac{\partial N(r, t)}{\partial t}=I(r)+D \nabla_{\perp}^{2} N-\gamma_{e}\left[N\left(1+\sum_{i=0,1} \sum_{j=x, y}\left|E_{i j}\right|^{2} \psi_{i j}^{2}(r)\right)-i n \sum_{i=0,1}\left(E_{i x} E_{i y}^{*}-E_{i y} E_{i x}^{*}\right) \psi_{i x}(r) \psi_{i y}(r)\right] \text {, } \\
& \frac{\partial n(r, t)}{\partial t}=-\gamma_{s} n+D \nabla_{\perp}^{2} n-\gamma_{e}\left[n \sum_{i=0,1} \sum_{j=x, y}\left|E_{i j}\right|^{2} \psi_{i j}^{2}(r)-i N \sum_{i=0,1}\left(E_{i x} E_{i y}^{*}-E_{i y} E_{i x}^{*}\right) \psi_{i x}(r) \psi_{i y}(r)\right],
\end{aligned}
$$

be obtained when this device is subject to two-frequency orthogonal optical injection. We also consider multitransverse-mode VCSELs subject to the same type of optical injection. Again, we obtain the periodic oscillations for this system. We show that the extra degree of freedom given by the multi-transverse-mode operation of the VCSEL is useful for enhancing the performance of the photonic microwave generation system. Two relative maxima of the microwave signal amplitude are obtained for two different values of the detuning between the lowest frequency injection and the where $\psi_{0 j}$ and $\psi_{1 j}$ are the modal profiles of the $j$-polarized $\mathrm{LP}_{01}$ and $\mathrm{LP}_{11}$ modes $(j=x, y)$ obtained by solving the Helmholtz equation [31], respectively, $E_{0 j}$ and $E_{1 j}$ are the modal amplitudes of these modes, $N(r, t)$ is the total carrier number, and $n(r, t)$ is the difference between the carrier numbers of the two magnetic sublevels. $\kappa_{r}$ is the relative loss of the $\mathrm{LP}_{11}$ mode with respect to the $\mathrm{LP}_{01}$ mode. That parameter determines the value of the injection current at which the $\mathrm{LP}_{11}$ mode begins lasing. $I(r)$ represents a uniform current injection over a circular disc of $6 \mu \mathrm{m}$ radius, that is, $I(r)=I$ if 
$r<6 \mu \mathrm{m}$, and $I(r)=0$, elsewhere. The normal gain normalized to the threshold gain $g_{i j}(i=0,1, j=x, y)$ and $g_{i j k}$ $(i=0,1 ; j k=x y, y x)$ are given by

$$
\begin{aligned}
g_{i j} & =\frac{\int_{0}^{\infty} N(r, t) \psi_{i j}^{2}(r) r \mathrm{~d} r}{\int_{0}^{\infty} \psi_{i j}^{2}(r) r \mathrm{~d} r}, \\
g_{i, j k} & =\frac{\int_{0}^{\infty} n(r, t) \psi_{i j}(r) \psi_{i k}(r) r \mathrm{~d} r}{\int_{0}^{\infty} \psi_{i j}^{2}(r) r \mathrm{~d} r} .
\end{aligned}
$$

In the two-frequency injection scheme, the VCSEL is optically injected by a master laser 1 (ML1) and a master laser 2 (ML2), with optical frequencies $\omega_{1}$ and $\omega_{2}\left(\omega_{1}<\omega_{2}\right)$, respectively. Two frequency detunings, $\Delta \omega_{1}=\omega_{1}-\omega_{\text {th }}$ and $\Delta \omega_{2}=\omega_{2}-\omega_{\text {th }}$, with respect to the central frequency between the two polarizations of the fundamental mode $\omega_{\text {th }}=\left(\omega_{0 x}+\omega_{0 y}\right) / 2$, appear in the equations. The injection terms only appear in the equations for $E_{0 y}$ and $E_{1 y}$, and hence linear optical injection in the $y$ direction is considered for both master lasers. In this way orthogonal optical injection is achieved if the free-running VCSEL emits only in the $x$-linear polarization, as it will be considered in this work. Optical injection terms are also characterized by the injection strengths, $\kappa_{i m}$, and the VCSEL roundtrip time, $\tau_{i n}=2 L / v_{g}$, where $v_{g}$ is the group velocity. The injection strengths $\kappa_{i m}(i=0,1$, $m=1,2$ ) are given by

$$
\kappa_{i m}=\left(\frac{1}{\sqrt{R}}-\sqrt{R}\right) \sqrt{\eta_{i n j}} \sqrt{P_{i n j, i m}}
$$

where $R$ is the output-mirror reflectivity, $\eta_{i n j}$ is the coupling efficiency of the injected light to the optical field in the laser cavity, and $P_{i n j i m}$ is the power injected in the $i$-transverse mode by the $m$-master laser [32]. Our initial choice of $\kappa_{i m}$ values in this work is given by what should be expected when light from a multi-transverse-mode VCSEL is injected into a similar device. $\kappa_{01}\left(\kappa_{12}\right)$ is related to the spatial overlap integral between the profiles of the fundamental modes (the higherorder modes) of master and slave lasers. Then a reasonable choice is $\kappa_{01}=\kappa_{12}=\kappa_{s}$. $\kappa_{02}\left(\kappa_{11}\right)$ is related to the spatial overlap integral between the profiles of the fundamental mode of the slave and the higher-order mode of the master (higher-

\begin{tabular}{|c|c|c|}
\hline Symbol & Value & Meaning of the Symbol \\
\hline$a$ & $6 \mu \mathrm{m}$ & Radius of the core region \\
\hline$L$ & $1 \mu \mathrm{m}$ & Length of the cavity \\
\hline$n_{\text {core }, x}$ & 3.5001025 & $\begin{array}{l}\text { Refractive index of the core region } \\
\text { in the } x \text { direction }\end{array}$ \\
\hline$n_{\text {core }, y}$ & 3.5 & $\begin{array}{l}\text { Refractive index of the core region } \\
\text { in the } y \text { direction }\end{array}$ \\
\hline$n_{\text {cladd }}$ & 3.41 & Refractive index of the cladding region \\
\hline$k$ & $300 \mathrm{~ns}^{-1}$ & Field decay rate \\
\hline$\alpha$ & 3 & Linewidth enhacement factor \\
\hline$\gamma_{e}$ & $0.55 \mathrm{~ns}^{-1}$ & Decay rate for the total carrier population \\
\hline$\gamma_{s}$ & $1000 \mathrm{~ns}^{-1}$ & Spin-flip relaxation rate \\
\hline$D$ & $10 \mathrm{~cm}^{2} \mathrm{~s}^{-1}$ & Diffusion coefficient \\
\hline$\beta$ & $10^{-5} \mathrm{~ns}^{-1}$ & Spontaneous emission coefficient \\
\hline$\gamma_{a}$ & $-0.3 \mathrm{~ns}^{-1}$ & Dichroism \\
\hline
\end{tabular}
order mode of the slave and the fundamental mode of the

Table 1. Device and Material Parameters master) VCSEL. In this way we initially choose $\kappa_{02}=\kappa_{11}=\kappa_{c}$. We call this parameter the crossed injection strength, because it represents the injection strength between transverse modes of different order. A more general choice of these parameters will be considered in Section 5 . The rest of the parameters that appear in the equations and their meanings are specified in the Table 1 . The frequency splitting between the orthogonal polarizations of the $\mathrm{LP}_{01}$ mode, $2 \gamma_{p 0} /(2 \pi)$, between the orthogonal polarizations of the $\mathrm{LP}_{11}$ mode, $2 \gamma_{p 1} /(2 \pi)$, and between the two transverse modes with the same polarization, $\gamma_{p}^{t r} /(2 \pi)$, are obtained from the calculation of the waveguide modes via the Helmholtz equation [13]. We have chosen the values of $n_{\text {core }, x}$, $n_{\text {core }, y}$, and $n_{\text {cladd }}$ in such a way that $2 \gamma_{p 0} /(2 \pi)=10 \mathrm{GHz}$ and $\gamma_{p}^{t r} /(2 \pi)=63 \mathrm{GHz}$. Spontaneous emission noise processes are modeled by the terms $\xi_{ \pm}$taken as complex Gaussian white noise sources of zero mean and delta-correlated in time. In the noise terms, the carrier distribution is integrated over the active region:

$$
\bar{N}=\frac{\int_{0}^{a} N(r, t) r \mathrm{~d} r}{a^{2}}, \quad \bar{n}=\frac{\int_{0}^{a} n(r, t) r \mathrm{~d} r}{a^{2}} .
$$

Time and space integration steps of $0.01 \mathrm{ps}$ and $0.12 \mu \mathrm{m}$, respectively, have been used. The boundary conditions for the carrier distribution are taken as $N(\infty, t)=0, n(\infty, t)=0$. The initial conditions correspond to a below-threshold stationary solution, i.e., to $I=0.1 . I_{\mathrm{th}}$, where $I_{\mathrm{th}}$ is the threshold current.

\section{SINGLE-TRANSVERSE-MODE VCSELS SUBJECT TO TWO-FREQUENCY ORTHOGONAL OPTICAL INJECTION}

Results corresponding to the dynamical evolution of a freerunning single-mode VCSEL are shown in Fig. 1(a). We have chosen a value $\kappa_{r}=3$ to assure fundamental transverse-mode operation. A rigorous determination of $\kappa_{r}$ should come out of the proper optical modelling of the VCSEL. Our simpler approach consists of choosing the value of $\kappa_{r}$ that gives the injection current at which the higher-order mode begins lasing appropriated to the type of VCSEL that we want to analyze. The laser is switched on at time $t>0$ with an $I=1.8 . I_{\text {th }}$ bias current value. The VCSEL emits in the steady state in the $x$ polarization with a single-peaked $\mathrm{RF}$ spectrum appearing at the relaxation oscillation frequency. The optical spectrum in the lowest part of Fig. 1(a) shows the $10 \mathrm{GHz}$ frequency separation between $\mathrm{LP}_{01, y}$ and $\mathrm{LP}_{01, x}$. We now present the results corresponding to the two-frequency orthogonal optical injection on that single-transverse-mode VCSEL. Results are given in terms of the separation between the frequencies of the two master lasers, $\Delta f=\left(\omega_{2}-\omega_{1}\right) /(2 \pi)$, the frequency detuning of ML1 with respect to the frequency of the $\mathrm{LP}_{01, y}$ mode, $\Delta \nu$, and the injection strength $\kappa_{s}$. In our calculations, except where stated otherwise, we consider a value of $\kappa_{c}=$ $\kappa_{s} / 2$ Orthogonal optical injection is applied for $t>4 \mathrm{~ns}$. We let the system evolve during an additional transient time of $10 \mathrm{~ns}$, above which data are collected in order to calculate $\mathrm{RF}$ and optical spectra. Figure 1(b) shows the results for a weak injection of $\kappa_{s}=10^{-5}$, with the frequency of ML1 just at the $\mathrm{LP}_{01, y}$ modal frequency. A similar time evolution to that of the free-running VCSEL is obtained. The effects of the orthogonal injection are clearer in the spectra. In the 

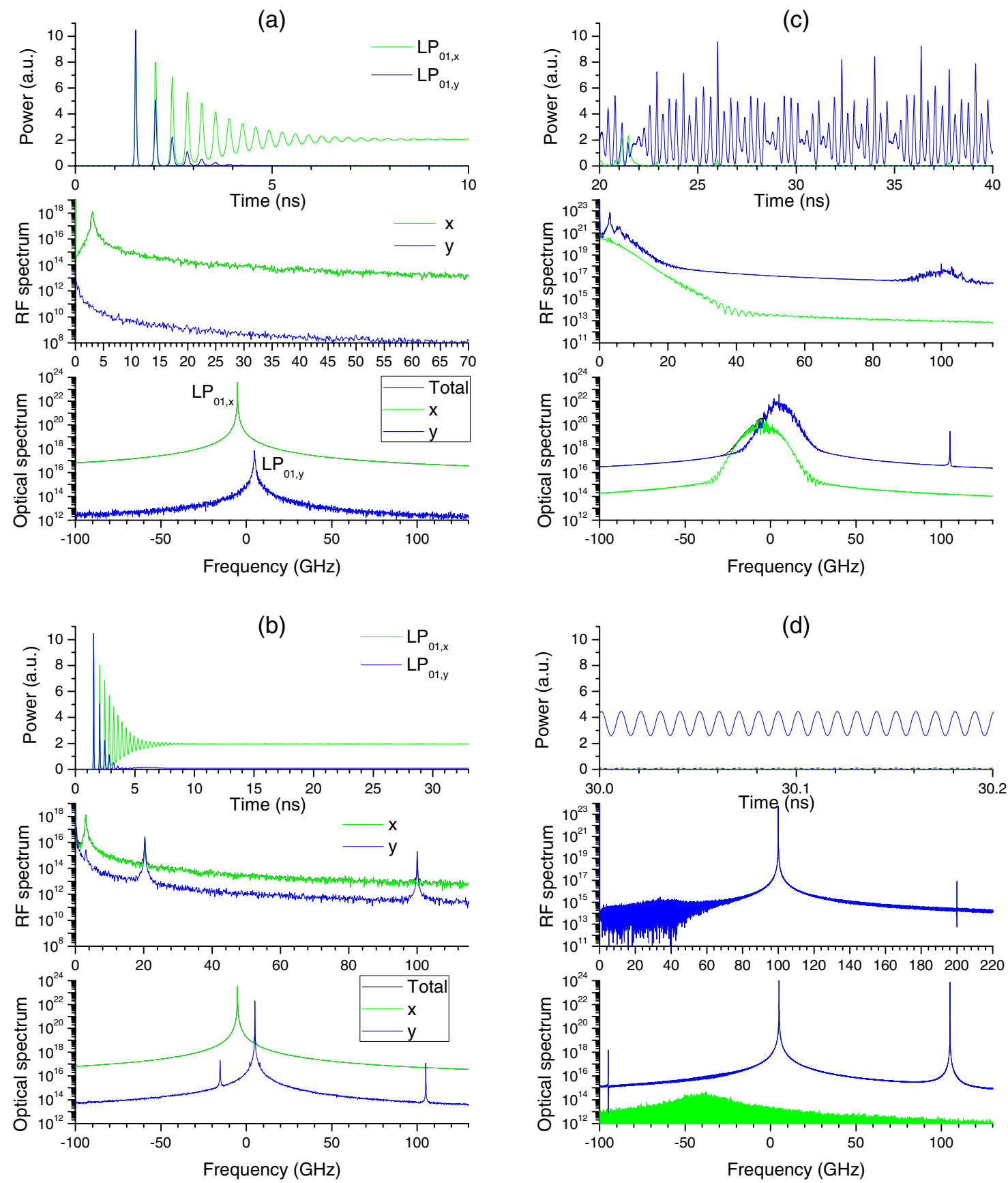

Fig. 1. (Color online) Temporal and spectral dynamics of the single-transverse-mode VCSEL when $\Delta f=100 \mathrm{GHz}$ and $\Delta \nu=0 \mathrm{GHz}$, with (a) no injection, (b) $\kappa_{s}=10^{-5}$, (c) $\kappa_{s}=2 \cdot 10^{-4}$, and (d) $\kappa_{s}=10^{-2}$. Upper row: time traces of the power of the polarized transverse modes. Middle row: RF spectra of the polarized powers. Lower row: optical spectra of the $x$-polarized (grey curve), $y$-polarized (dark grey curve), and total power.

$y$-polarized optical spectrum the peak corresponding to the $\mathrm{LP}_{01, y}$ mode (at $5 \mathrm{GHz}$ ) is enhanced, and a symmetric peak with respect to the $\mathrm{LP}_{01 . x}$ frequency appears at $-15 \mathrm{GHz}$. The origin of this peak is possibly due to wave-mixing dynamics $[32,33]$. The peak corresponding to the frequency of ML2 also appears at $105 \mathrm{GHz}$. The frequency separation between the first two peaks in the $y$-polarized spectrum is $20 \mathrm{GHz}$, which is the value of the frequency at which the RF spectrum of the $y$-polarization develops an additional peak. A peak also appears at $100 \mathrm{GHz}$, which is precisely the frequency separation between ML2 and ML1. An irregular dynamics is obtained when increasing $\kappa_{s}$, as illustrated in Fig. 1(c). PS has been achieved, because almost all the power is $y$-polarized; the $x$-polarization only appears eventually with small power. This irregular dynamics is also characterized by the broadened optical and RF spectra. A further increase of $\kappa_{s}$ leads to sinusoidal periodic oscillations for the power of the $\mathrm{LP}_{01, y}$ mode. The frequency of this sinusoidal modulation is $\Delta f$. The optical spectrum consists in two well-defined peaks at the ML1 and ML2 optical frequencies, and the RF spectrum has a very narrow peak at $\Delta f$, the frequency separation between ML2 and ML1. This situation is similar to the so-called "double injection locking" experimentally observed in a singlemode DFB laser subject to dual-beam injection [28] and 
theoretically described using a single-mode rate equation model $[27,29]$. The dynamical evolution is also similar to that observed when two-frequency parallel optical injection is applied to a single-transverse-mode VCSEL [30]. In [28-30] a linearly polarized single mode laser is subject to a parallel polarized two-frequency optical injection. The main difference in our results with respect to those in [28-30] lies in the consideration of the additional degree of freedom corresponding to the polarization: periodic oscillations can also be achieved when using orthogonally polarized two-frequency injection.

Wide tunability of the microwave signal generated by twofrequency parallel optical injection on single-mode semiconductor lasers was shown in [28-30]. Figure 2 shows that our system also displays wide tunability. Results are shown for several values of $\Delta f$, from below the transverse mode separation [Fig. 2(a)], at the transverse mode separation [Fig. 2(b)], and well above the transverse mode separation [Fig. 2(c)]. In all the cases, well-defined sinusoidal time traces are obtained for the power of the $\mathrm{LP}_{01, y}$ mode. The amplitude of the oscillations decreases as $\Delta f$ increases. All the $\mathrm{RF}$ spectra show a narrow peak at the $\Delta f$ frequency, even at the very large values simulated in Fig. 2(c), well beyond the microwave range.

\section{MULTI-TRANSVERSE-MODE VCSELS SUBJECT TO TWO-FREQUENCY ORTHOGONAL OPTICAL INJECTION}

Results corresponding to two-frequency orthogonal optical injection on a multi-transverse-mode VCSEL are presented in this section. The multi-transverse-mode VCSEL that is simulated is exactly the same as that of the previous section, but with the parameter $\kappa_{r}$ changed to a smaller value for which both transverse modes have rather similar losses. Results obtained for $\kappa_{r}=1.022$ for a free-running multi-mode VCSEL $\left(\kappa_{s}=\kappa_{c}=0\right)$ are plotted in Fig. 3(a). The solitary VCSEL shows a steady state in which the $\overline{\mathrm{LP}}_{01, x}$ and $\mathrm{LP}_{11, x}$ modes are excited. The steady-state total power in Fig. 3(a) is 1.7, a similar value to the value of 2 that was obtained for Fig. 1(a). The power in $\mathrm{LP}_{11, x}$ is only slightly larger than that of $\mathrm{LP}_{01, x}$. Figure $3(\mathrm{a})$ shows that the $x$-polarized RF spectrum has two peaks (at 1 and $3 \mathrm{GHz}$ ) that appear due to the multitransverse-mode emission of the VCSEL [34-36]. Linearization of the stochastic rate equations around an analytical steadystate solution has shown that the relative intensity noise in multi-transverse modes have resonance peaks that appear at frequencies that correspond to the relaxation oscillation frequencies of the multimode laser [36]. The multimode character of the system is also clear from the optical spectra of Fig. 3(a), in which the peaks corresponding to $\mathrm{LP}_{01, x}$ and $\mathrm{LP}_{11, x}$ are excited with similar values. The separation between $x$-polarized transverse modes, $\mathrm{LP}_{11, x}$ and $\mathrm{LP}_{01, x}$, is around $63 \mathrm{GHz}$, a value similar to the separation between $\mathrm{LP}_{11, y}$ and $\mathrm{LP}_{01, y}$.

Figures $3(\mathrm{~b})-3(\mathrm{~d})$ show the results of the multi-transversemode VCSEL subject to two-frequency orthogonal optical injection. The levels of the injection strength, $\Delta f$, and $\Delta \nu$ are equal to those considered in the single-transverse-mode case (Fig. 1). In this way we can compare the performance of microwave generating systems using single- and multitransverse-mode VCSELs. Figure 3(b) shows the results for a weak injection of $\kappa_{s}=10^{-5}$, with the frequency of ML1 just at the $\mathrm{LP}_{01, y}$ mode frequency. A rather similar temporal evolution to that found in free-running VCSELs is obtained with some new characteristic features. Some power appears in the $\mathrm{LP}_{01, y}$ mode, with a corresponding decrease in the power of the $\mathrm{LP}_{01, x}$ mode. The optical spectrum shows that the peak corresponding to the $\mathrm{LP}_{01, y}$ mode (at $5 \mathrm{GHz}$ ) is enhanced and a small symmetric peak with respect to the $\mathrm{LP}_{01, x}$ frequency appears at $-15 \mathrm{GHz}$. Two other strong peaks appear in that spectrum: the first, near $70 \mathrm{GHz}$, appears at the frequency of the $\mathrm{LP}_{11, y}$ mode, and the second one, near $105 \mathrm{GHz}$, appears at the ML2 frequency. Frequency differences between those peaks explain the multi-peaked structure of the $y$-polarized $\mathrm{RF}$ spectrum. A regular multimode dynamics, involving the $\mathrm{LP}_{01, y}$ and $\mathrm{LP}_{11, x}$ modes, is obtained when increasing $\kappa_{s}$, as illustrated in Fig. 3(c), in contrast to the irregular dynamics of Fig. 1(c). A complete PS has not been achieved yet, in contrast to Fig. 1(c), for which the increase of injection strength
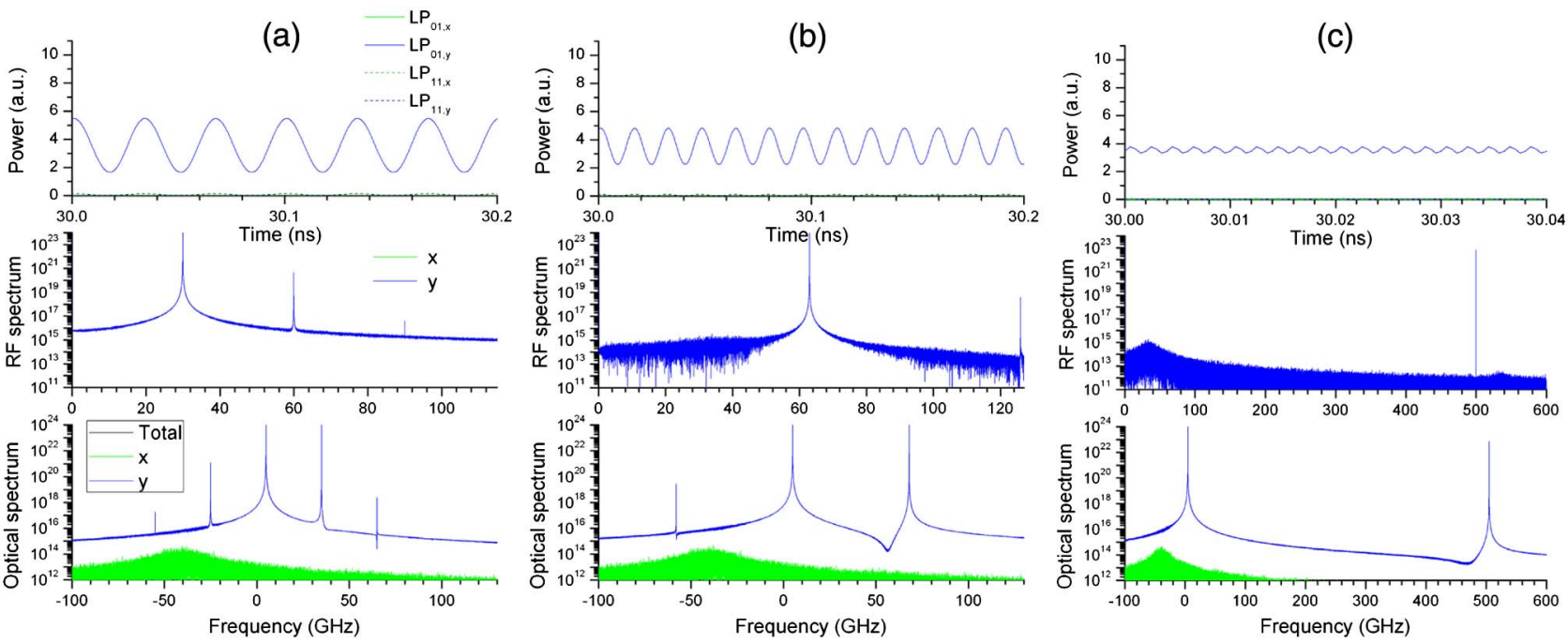

Fig. 2. (Color online) Temporal and spectral dynamics of the single-transverse-mode VCSEL when $\Delta f=30,63$, and $500 \mathrm{GHz}$ and $\Delta \nu=0 \mathrm{GHz}$ $\kappa_{s}=10^{-2}$. Upper row: time traces of the power of the polarized transverse modes. Middle row: RF spectra of the polarized powers. Lower row: optical spectra of the $x$-polarized (grey curve), $y$-polarized (dark grey curve), and total power. 

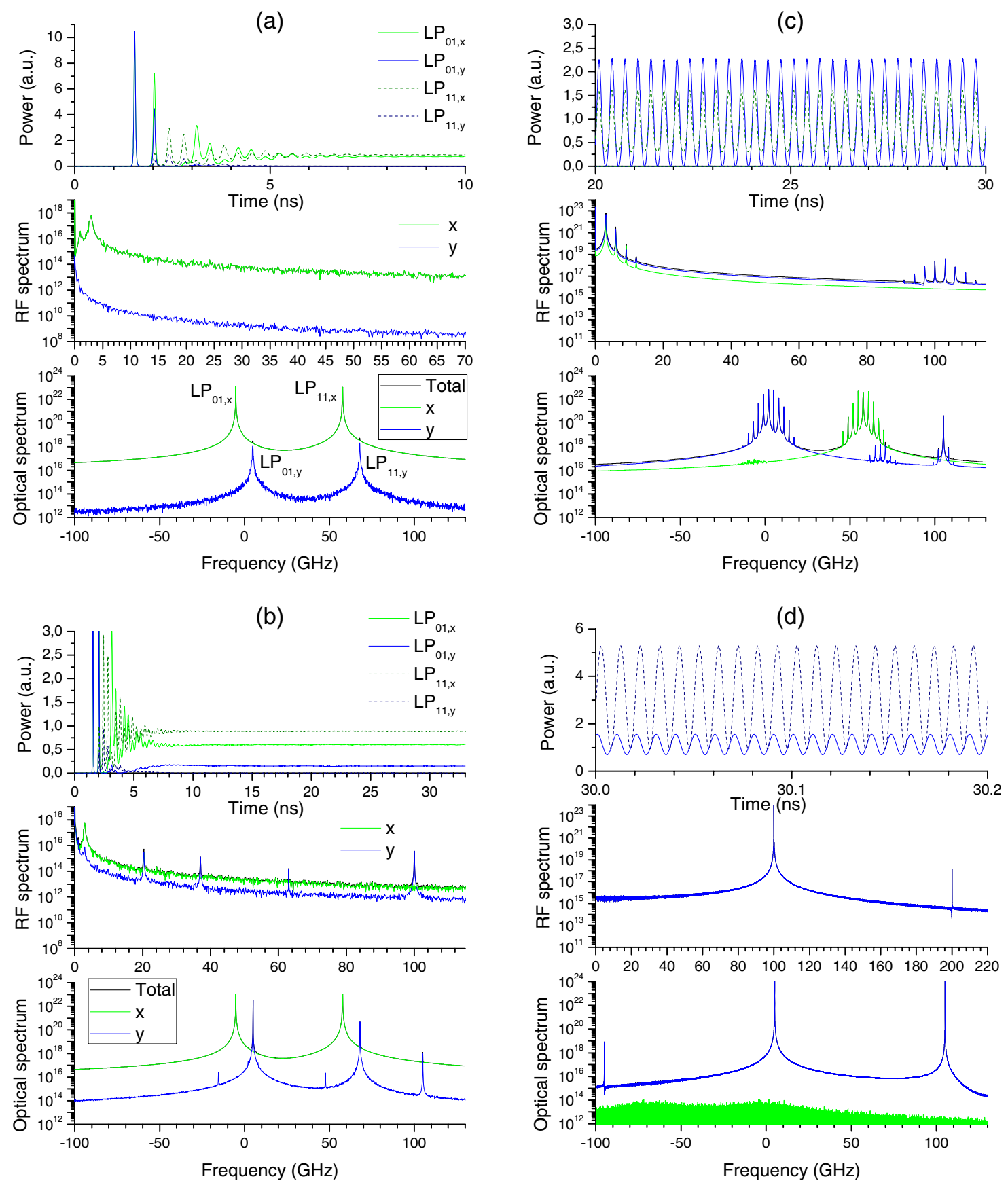

Fig. 3. (Color online) Temporal and spectral dynamics of the multi-transverse-mode VCSEL when $\Delta f=100 \mathrm{GHz}$ and $\Delta \nu=0 \mathrm{GHz}$, with (a) no injection, (b) $\kappa_{s}=10^{-5}$, (c) $\kappa_{s}=2 \cdot 10^{-4}$, and (d) $\kappa_{s}=10^{-2}$. Upper row: time traces of the power of the polarized transverse modes. Middle row: RF spectra of the polarized powers. Lower row: optical spectra of the $x$-polarized (grey curve), $y$-polarized (dark grey curve), and total power.

resulted in the disappearance of the $x$-polarized power. Figure 3(d) shows the case in which, similar to Fig. 1(d), periodic oscillations are obtained. Again, two well-defined peaks at the frequencies of ML1 and ML2 are observed in the $y$ polarized optical spectrum, resulting in an RF spectrum with a narrow peak at the $\Delta f$ frequency. Complete PS has been obtained. Figure 3(d) also shows that the high-order transverse mode $\mathrm{LP}_{11, y}$ is excited with a much larger amplitude than that of the $\mathrm{LP}_{01, y}$ mode. The amplitude of the power time trace of the $\mathrm{LP}_{11, y}$ mode is 5.1 times larger than that corresponding to the $\mathrm{LP}_{01, y}$ mode, with a $\sim \pi / 2 \mathrm{rad}$ phase difference. The variation of the total power is nearly sinusoidal, as shown in the RF spectrum of Fig. 3(d). The amplitude of the total power is much larger than that obtained with the similar single-transverse-mode VCSEL case illustrated in Fig. 1(d). This shows that the amplitude of the microwave signal generated by two-frequency orthogonal optical injection is enhanced if multi-transverse-mode operation in the VCSEL is considered instead of single-transverse-mode operation. These results are similar to the results obtained for twofrequency parallel optical injection [30].

We now analyze the effect of the frequency detuning, $\Delta \nu$, on the dynamics of the system. We focus on situations in which periodic oscillations are achieved. Figures $\underline{4(a)}-\underline{4(c)}$ 
(a)
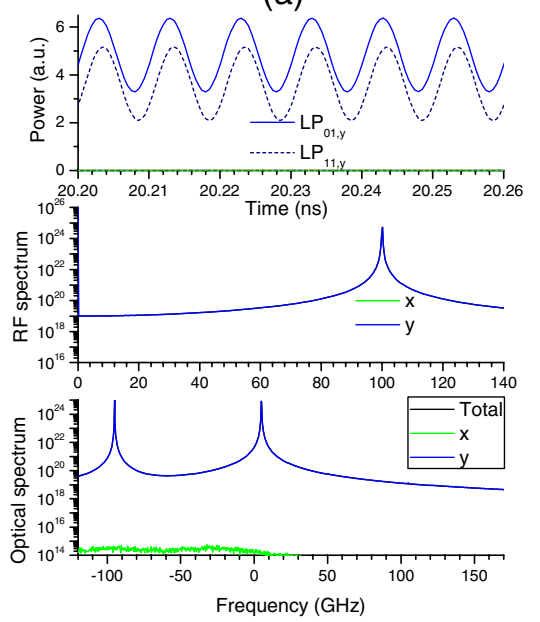

(b)

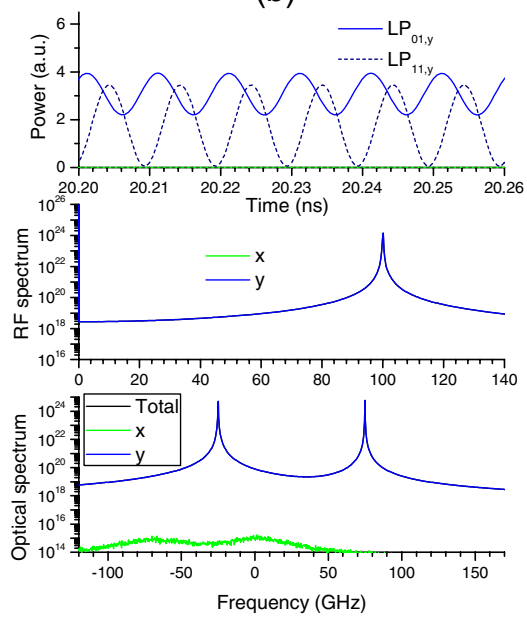

(c)

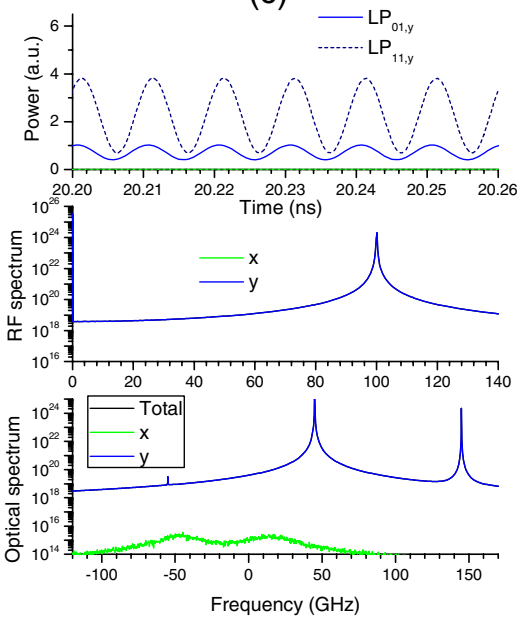

Fig. 4. (Color online) Temporal and spectral dynamics of the multi-transverse-mode VCSEL when $\Delta f=100 \mathrm{GHz}$ and $\kappa_{s}=10^{-2}$ when (a) $\Delta \nu=-100 \mathrm{GHz}$, (b) $\Delta \nu=-30 \mathrm{GHz}$, and (c) $\Delta \nu=40 \mathrm{GHz}$. Upper row: time traces of the power of the polarized transverse modes. Middle row: RF spectra of the polarized powers. Lower row: optical spectra of the $x$-polarized (grey curve), $y$-polarized (dark grey curve), and total power.

show the results obtained when $\Delta \nu$ is $-100,-30$, and $40 \mathrm{GHz}$, respectively. Similar to Fig. 3(d), RF spectra show a welldefined peak at $\Delta f=100 \mathrm{GHz}$. This shows that periodic oscillations are obtained over a very wide $\Delta \nu$ range. This is also illustrated in Fig. 5, in which the peak-to-peak amplitudes of the total power, $\bar{A}_{\text {tot }}$, and of the $\mathrm{LP}_{01 . y}$ and $\mathrm{LP}_{11, y}$ modes, $A_{01}$ and $A_{11}$, respectively, are plotted as a function of $\Delta \nu$. Figure 4(a) shows the case in which the frequency of ML2 coincides with the $\mathrm{LP}_{01, y}$ modal frequency. Sinusoidal time traces of $\mathrm{LP}_{01, y}$ and $\mathrm{LP}_{11, y}$ modes with similar amplitudes are obtained. The very small phase difference between these $(\sim 0.1 \pi \mathrm{rad})$ causes large values of $A_{\text {tot }}$, as is also shown in Fig. 5. In fact, the maximum value of $A_{\text {tot }}$ is obtained at $\Delta \nu=-105 \mathrm{GHz}$. Figure 5 also shows that $A_{\text {tot }}$ has a relative minimum and another relative maximum at $\Delta \nu=-30$ and $0 \mathrm{GHz}$, respectively. Figure 4(b) illustrates the dynamics obtained at that relative minimum. The expected shift in the $y$-polarized optical spectrum with respect to Fig. 4(a) is observed, because now the light emitted by ML1 is injected at $-30 \mathrm{GHz}$ with respect to the free-running $\mathrm{LP}_{01, y}$ frequency. The amplitude of the power time trace of the $\mathrm{LP}_{11, y}$ mode is 1.9 times larger than that corresponding to the $\mathrm{LP}_{01, y}$ mode, with a phase difference of $0.62 \pi$ rad. Figure $4(\mathrm{~b})$ also shows that the power time trace of the $\mathrm{LP}_{11, y}$ mode vanishes periodically. In this way the relative minimum of $A_{\text {tot }}$ is obtained at this value of $\Delta \nu$. The second relative maximum of $A_{\text {tot }}$ appears at $\Delta \nu=0 \mathrm{GHz}$, which is when the frequency of ML1 coincides with the $\mathrm{LP}_{01, y}$ modal frequency. The corresponding dynamical evolution was already illustrated in Fig. 3(d). Figure 4(c) shows the dynamical evolution obtained when $\Delta \nu=40 \mathrm{GHz}$. A small phase difference $(\sim 0.1 \pi \mathrm{rad})$ between time traces of $\mathrm{LP}_{01, y}$ and $\mathrm{LP}_{11, y}$ modes and a large amplitude of the power of $\mathrm{LP}_{11, y}$ mode are obtained.

Figure 5 shows that $A_{\text {tot }}$ has two relative maxima, at -105 and $0 \mathrm{GHz}$, that are obtained when the frequencies of ML2 and ML1 are very close to the $\mathrm{LP}_{01, y}$ modal frequency, respectively. $A_{01}$ has only a maximum that is obtained when the frequency of ML2 coincides with the $\mathrm{LP}_{01, y}$ frequency. $A_{11}$ has two relative maxima, at $\Delta \nu=-115$ and $-5 \mathrm{GHz}$. The maximum value of $A_{\text {tot }}$ at $\Delta \nu=-105 \mathrm{GHz}$ is approximately the sum of the relative maxima of $A_{01}$ and $A_{11}$, because the phase difference between time traces of the $\mathrm{LP}_{01, y}$ and $\mathrm{LP}_{11, y}$ modes is very small. The maximum value of $A_{\text {tot }}$ at $\Delta \nu=0 \mathrm{GHz}$ is given mainly by the contribution of the largest relative maximum of $A_{11}$. Figure 5 also shows the dependence of the ratio $A_{11} / A_{01}$ on $\Delta \nu$. This ratio is near 1 when $-125 \mathrm{GHz}<\Delta \nu<$ $-70 \mathrm{GHz}$, which is when the frequency of ML2 is near the $\mathrm{LP}_{01, y}$ frequency. $A_{11} / A_{01}$ has a maximum value of 5.3 when $\Delta \nu=5 \mathrm{GHz}$, which is when the frequency of ML1 is very near to the $\mathrm{LP}_{01, y}$ frequency. Large values of $A_{11} / A_{01}$ are extended over a wide range of positive $\Delta \nu$.

\section{DISCUSSION}

We now discuss the physical reasons for the high-frequency oscillations of the power of the transverse modes. Figure 6 shows the dynamical evolution of the multi-transverse-mode VCSEL subject to two-frequency orthogonal optical injection

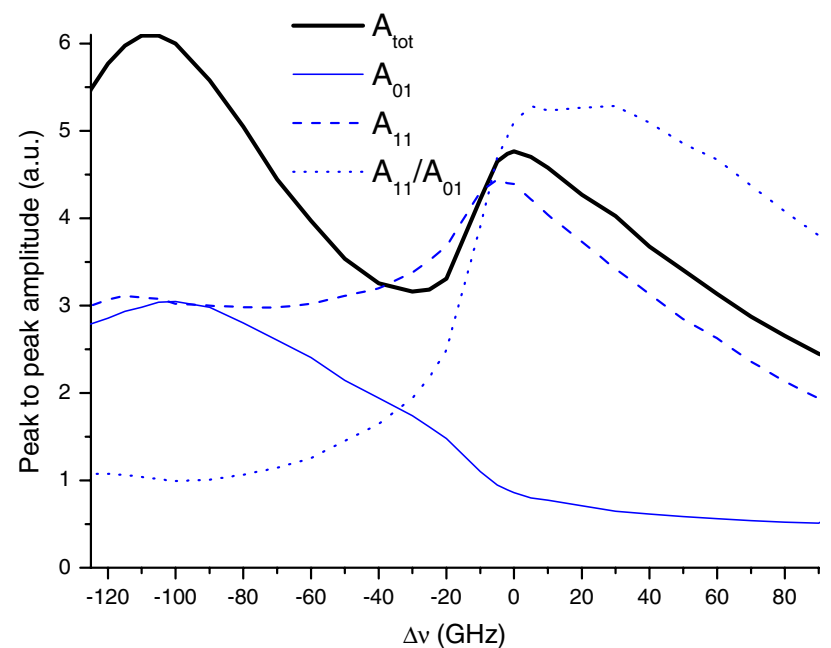

Fig. 5. (Color online) Peak-to-peak amplitude of the total power (thick solid curve), the $\mathrm{LP}_{01, y}$ power (thin solid curve), and the $\mathrm{LP}_{11, y}$ power (dashed curve) as a function of $\Delta \nu$. The ratio between $A_{11}$ and $A_{01}$ is also plotted with a dotted curve. In this figure $\Delta f=100 \mathrm{GHz}$ and $\kappa_{\mathrm{s}}=10^{-2}$ 

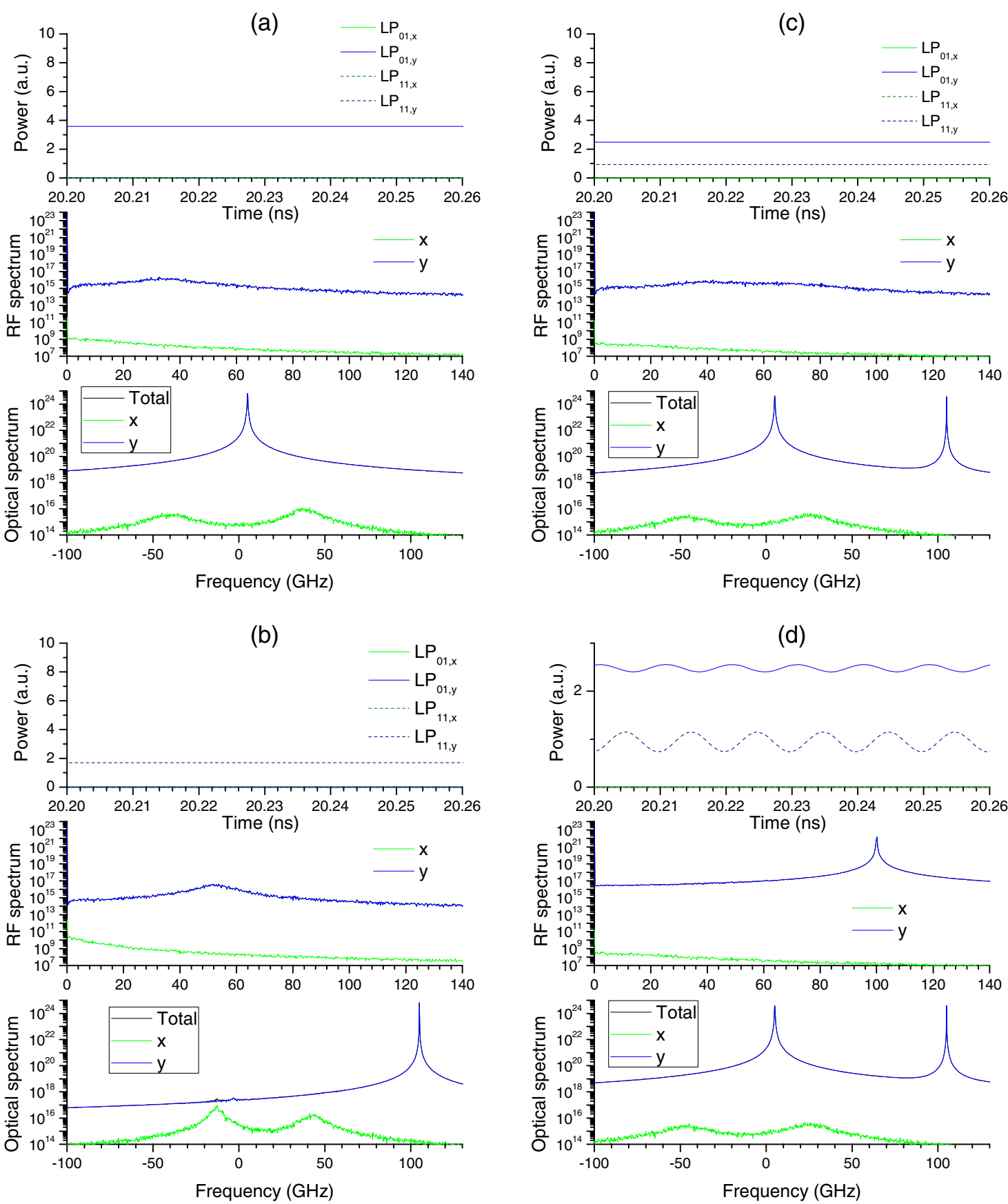

Fig. 6. (Color online) Temporal and spectral dynamics of the multi-transverse-mode VCSEL when $\Delta f=100 \mathrm{GHz}$ and $\Delta \nu=0 \mathrm{GHz}$, with (a) $\kappa_{01}=10^{-2}, \quad \kappa_{12}=\kappa_{02}=\kappa_{11}=0, \quad(\mathrm{~b}) \kappa_{12}=10^{-2}, \quad \kappa_{01}=\kappa_{02}=\kappa_{11}=0, \quad(\mathrm{c}) \kappa_{01}=\kappa_{12}=10^{-2}, \quad \kappa_{02}=\kappa_{11}=0$, and (d) $\kappa_{01}=\kappa_{12}=10^{-2}$, $\kappa_{02}=\kappa_{11}=5 \cdot 10^{-4}$. Upper row: time traces of the power of the polarized transverse modes. Middle row: RF spectra of the polarized powers. Lower row: optical spectra of the $x$-polarized (grey curve), $y$-polarized (dark grey curve), and total power.

under different injection situations. Figure $\underline{6(a)}$ shows the dynamics when there is only injection from ML1 and no coupling to the $\mathrm{LP}_{11, y}$ mode is considered $\left(\kappa_{01}=10^{-2}\right.$, $\kappa_{12}=\kappa_{02}=\kappa_{11}=0$ ). All the power is emitted with a constant value in the $\mathrm{LP}_{01, y}$ mode, and a flat $\mathrm{RF}$ spectrum is obtained. The optical spectrum consists in a single peak at the ML1 frequency. The situation when there is only injection from ML2 and no coupling to the $\mathrm{LP}_{01, y}$ mode is considered $\left(\kappa_{12}=10^{-2}\right.$, $\left.\kappa_{01}=\kappa_{02}=\kappa_{11}=0\right)$ is illustrated in Fig. 6(b). The optical spectrum has a single peak at the ML2 frequency, only the $\mathrm{LP}_{11, y}$ mode is excited with a constant power, and the $\mathrm{RF}$ spectrum is flat. Figure 6(c) illustrates the dynamics when injection from ML1 and $\overline{M L 2}$ is applied $\left(\kappa_{01}=\kappa_{12}=10^{-2}\right)$ in such a way that the crossed injection strengths vanish $\left(\kappa_{02}=\kappa_{11}=0\right)$. Now the optical spectrum has two peaks at the ML1 and ML2 frequencies, both $\mathrm{LP}_{01, y}$ and $\mathrm{LP}_{11, y}$ modal powers are excited with constant values, and the RF spectrum remains flat. The physical origin of the high-frequency oscillations lies in the nonzero values of the crossed injection

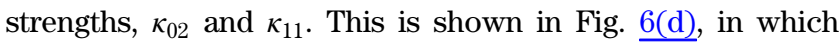
very small values of these parameters, $\kappa_{02}=\overline{\kappa_{11}}=5 \cdot 10^{-4}$, have been considered. These values are enough for the RF 
spectrum to develop a peak at the $\Delta f$ frequency with the corresponding oscillation of the power of both transverse modes.

In our previous calculations, we have assumed that the crossed injection strength parameter, $\kappa_{c}$, is equal for $\mathrm{LP}_{01, y}$ injected into $\mathrm{LP}_{11, y}$ and for $\mathrm{LP}_{11, y}$ injected into $\mathrm{LP}_{01, y}$ modes, that is, $\kappa_{c}=\kappa_{02}=\kappa_{11}$. Also, we have assumed that $\kappa_{01}=\kappa_{12}$ We now consider the more general case of asymmetric injection strengths for which $\kappa_{02}$ can be different from $\kappa_{11}$ and $\kappa_{01}$ can be different from $\kappa_{12}$. Figure 7 shows the peak-to-peak amplitude of the total power when $\kappa_{01}=10^{-2}$ as a function of $\kappa_{02}$ for different values of the other injection strengths, $\kappa_{11}$ and $\kappa_{12}$.
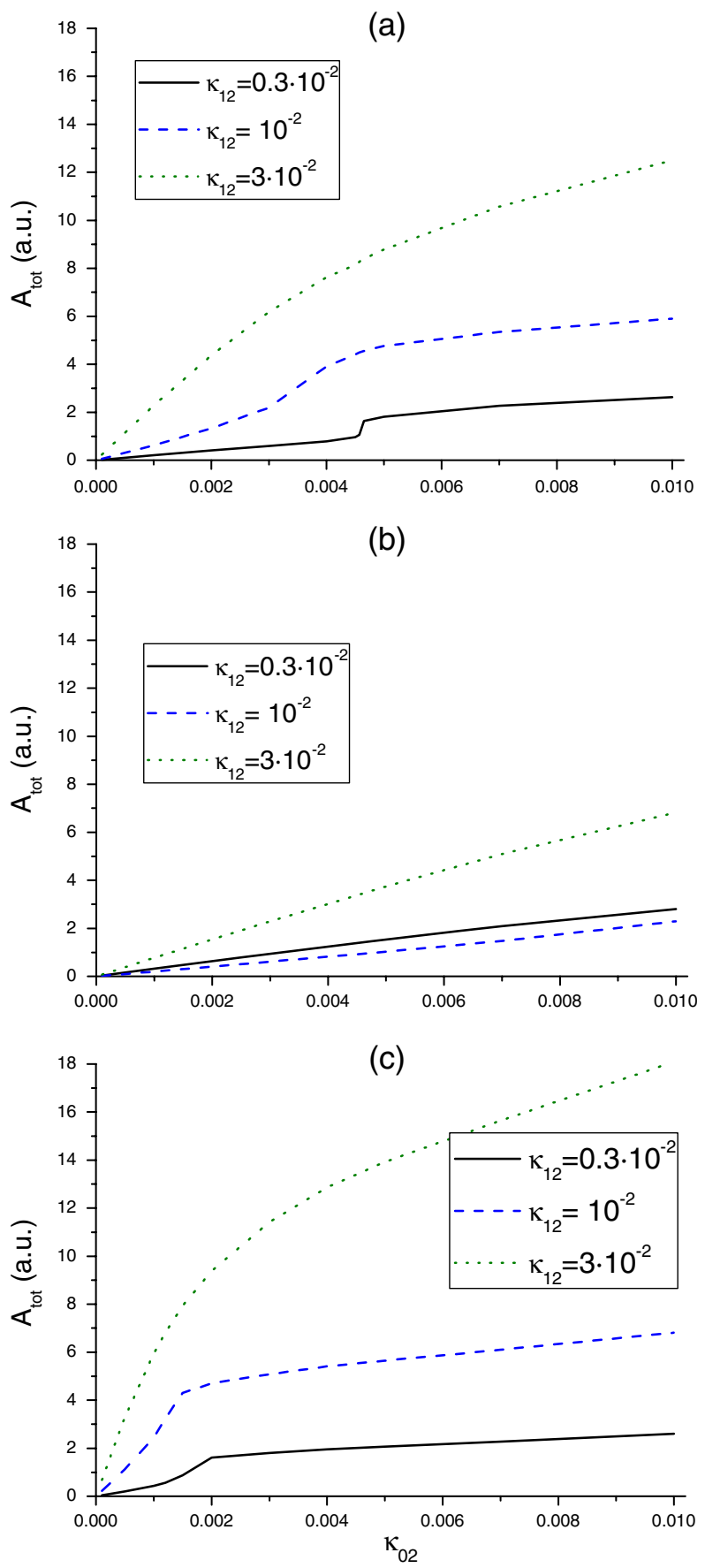

Fig. 7. (Color online) Peak-to-peak amplitude of the total power as a function of $\kappa_{02}$ when $\Delta f=100 \mathrm{GHz}, \Delta \nu=0 \mathrm{GHz}, \kappa_{01}=10^{-2}$, and (a) $\kappa_{11}=\kappa_{02}$, (b) $\kappa_{11}=0.3 \kappa_{02}$, and (c) $\kappa_{11}=3 \kappa_{02}$.
Figures $\underline{7(\mathrm{a})}-\underline{7(\mathrm{c})}$ illustrate the cases in which $\kappa_{02}$ is equal to, larger than, and smaller than $\kappa_{11}$, respectively. For all the cases considered in this figure, a sinusoidal evolution of the total power at the $\Delta f$ frequency, with an increasing value of $A_{\text {tot }}$ as $\kappa_{02}$ is increased, has been observed. $A_{\text {tot }}$ increases linearly with $\kappa_{02}$ providing that $\kappa_{02}$ is small enough. Also in agreement with the discussion of Fig. $\underline{6}, A_{\text {tot }} \rightarrow 0$ as $\kappa_{02} \rightarrow 0$. The dashed curve of Fig. $7($ a) illustrates the case of $\kappa_{02}=\kappa_{11}=\kappa_{c}, \kappa_{01}=\kappa_{12}=\kappa_{s}$ like in our previous calculations, but without the restriction of $\kappa_{c}=\kappa_{s} / 2$. Figure 7(a) also shows that larger $A_{\text {tot }}$ amplitudes are obtained as $\kappa_{12}$ increases. The small step observed in Fig. $\underline{7(a)}$ for $\kappa_{12}=$ $0.3 \cdot 10^{-2}$ is due to a sudden change of the phase difference between the power time series of both transverse modes that appears when $\kappa_{02}=0.0046$, similar to steps found in [30]. Figure $7(\mathrm{~b})$ illustrates a situation similar to that of Fig. $\overline{(\mathrm{a})}$, but with $\kappa_{11}=0.3 \kappa_{02}$. $A_{\text {tot }}$ increases linearly with $\kappa_{02}$ for the whole range of variation of $\kappa_{02}$, because smaller crossed injection strength values lead to that linear variation. Figure $7(\mathrm{~b})$ also shows that there is not a monotonous variation of $\overline{A_{\text {tot }}}$ with $\kappa_{12}$, because their values decreases when $\kappa_{12}$ changes from $0.3 \cdot 10^{-2}$ to $10^{-2}$. Figure $7(\mathrm{c})$ illustrates the situation when $\kappa_{11}=3 \kappa_{02}$, that is, with larger crossed injection strengths. These values produce larger $A_{\text {tot }}$ values and narrower linear regions than those of Figs. 7(a) and 7(b).

Rigorous evaluation of $k_{i m}$ parameters would involve the calculation of the spatial overlaps between electric fields coming from the master lasers and the electric fields corresponding to the transverse modes of the slave VCSEL. This calculation would be desirable, but it is beyond the scope of this manuscript. Taking into account the large diversity of values that $k_{01}, k_{12}, k_{02}$, and $k_{11}$ parameters can take, we have obtained most of our results for the case in which two similar VCSELs are considered, for which $k_{01}=k_{12}=k_{s}$ and $k_{02}=$ $k_{11}=k_{c}$ seem to be a reasonable approximation. Also, consideration of two similar VCSELs would mean having an upper limit for the injected power. Figure 7 has shown the amplitude of the periodic oscillations without the restrictions previously mentioned. Periodic oscillations with appreciable amplitudes are obtained for all the considered injection strengths, providing that $k_{02}$ is not close to zero. This means that periodic oscillations would be obtained despite the upper limit for the injected power, providing that $k_{02}$ is not close to zero.

We now discuss the enhancement in the microwave signal generation induced by the multi-transverse-mode operation of the VCSEL and the tunability of our system. Figure $\underline{8}$ shows $A_{\text {tot }}$ as a function of the frequency difference between ML2 and ML1, which is the frequency of the generated microwaves, $\Delta f$. Results for single- and multi-transverse-mode VCSELs are included. Also, results for two injection strength levels are shown. Again, all the cases reported in this figure have an almost sinusoidal variation of the total power. Peak-to-peak amplitudes decrease as $\Delta f$ increases. An increase of the injection strength $\kappa_{s}$ leads to larger peak-to-peak amplitudes for both single- and multi-transverse-mode cases. For all the values of $\Delta f$ and $\kappa_{s}$, the amplitude of the oscillation obtained with multi-transverse-mode VCSELs is larger than that obtained for single-transverse-mode VCSELs. The ratio between both amplitudes varies between 2 and 2.6 for the cases considered in Fig. 8. The ratio value of 2.6 is obtained for $\kappa_{s}=10^{-2}$ and $\Delta f=6 \overline{5} \mathrm{GHz}$, in which the value of $\Delta f$ is quite similar to the 


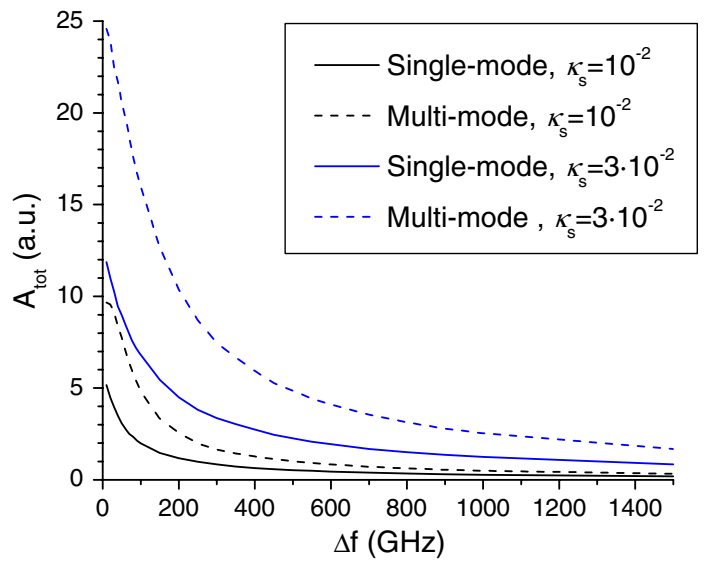

Fig. 8. (Color online) Peak-to-peak amplitude, $A_{\text {tot }}$, of the total power as a function of the generated frequency, $\Delta f$. In this figure $\Delta \nu=0 \mathrm{GHz}$ and $\kappa_{c}=\kappa_{s} / 2$.

transverse mode separation. This indicates that the maximum enhancement is obtained when the optical frequencies of ML1 and ML2 coincide with the frequencies of the free-running $\mathrm{LP}_{01, y}$ and $\mathrm{LP}_{11, y}$ modes, respectively.

Figure $\underline{8}$ also shows that the largest variations of peakto-peak amplitudes as $\Delta f$ changes occur in the microwave region $(<300 \mathrm{GHz})$. In the graph we have also considered a $\Delta f$ range that goes beyond the microwave range. In this region the generated radiation has an appreciable amplitude that increases as $\kappa_{s}$ is increased. The amplitude of this modulation slightly decreases when $\Delta f$ is in the THz region. Wide tuning ranges, extended into the $\mathrm{THz}$ band, are therefore obtained in our system. A more realistic study of the extention into the $\mathrm{THz}$ band would require the inclusion in the model of the dynamical evolution of the macroscopic polarization of the quantum well media, as is done, for instance, in [37]. Further study using this improved model would be desirable in order to verify that tuning ranges of the order of terahertz can be achieved using our system. In our simulations we have considered a very high value of the spin-flip relaxation rate $\left(\gamma_{s}=1000 \mathrm{GHz}\right)$. This value has been chosen because the best agreement with previous experimental results for a similar VCSEL subject to optical feedback [38] was obtained with that value of $\gamma_{s}$. Future work is also planned to investigate the influence of smaller values of this parameter, and hence of the spin of the carriers on our results. The results contained in Fig. 8 are very similar to those reported in [30] for parallel optical injection. When two-frequency parallel optical injection is considered, the maximum frequency of the generated microwave signals can be substantially increased if multimode VCSELs are used instead of single-mode VCSELs [30]. The fact that our results are very similar to those of [30] indicates that the previous result also holds when orthogonal optical injection is considered.

VCSEL dynamics under dual optical injection is much more complex than those found in a VCSEL subject to a single injection [27]. Several example of those dynamics and of their dependence on frequency detuning are shown in Fig. 9 when $\Delta f=100 \mathrm{GHz}$ and $\kappa_{s}=2 \cdot 10^{-4}$. Figure $9(\mathrm{a})$ shows that inphase sinusoidal oscillations of $\mathrm{LP}_{01, x}$ and $\mathrm{LP}_{11, x}$ and a notsinusoidal periodic response of the $\mathrm{LP}_{01, y}$ power are found for $\Delta \nu=-5 \mathrm{GHz}$. The situation for $\Delta \nu=0$ was already illustrated in Fig. 3(c). In that case in-phase oscillations were also found, but for different modes, $\mathrm{LP}_{01, y}$ and $\mathrm{LP}_{11, x}$. Figure $\underline{9(\mathrm{~b})}$ shows that for $\Delta \nu=5 \mathrm{GHz}$ the modes that participate in the dynamics are the same as those found when $\Delta \nu=-5 \mathrm{GHz}$. However, Fig. 9(b) illustrates a more complex dynamical evolution, because fast and slow oscillations of the modal powers are found. Fast oscillations are modulated by a slow periodic
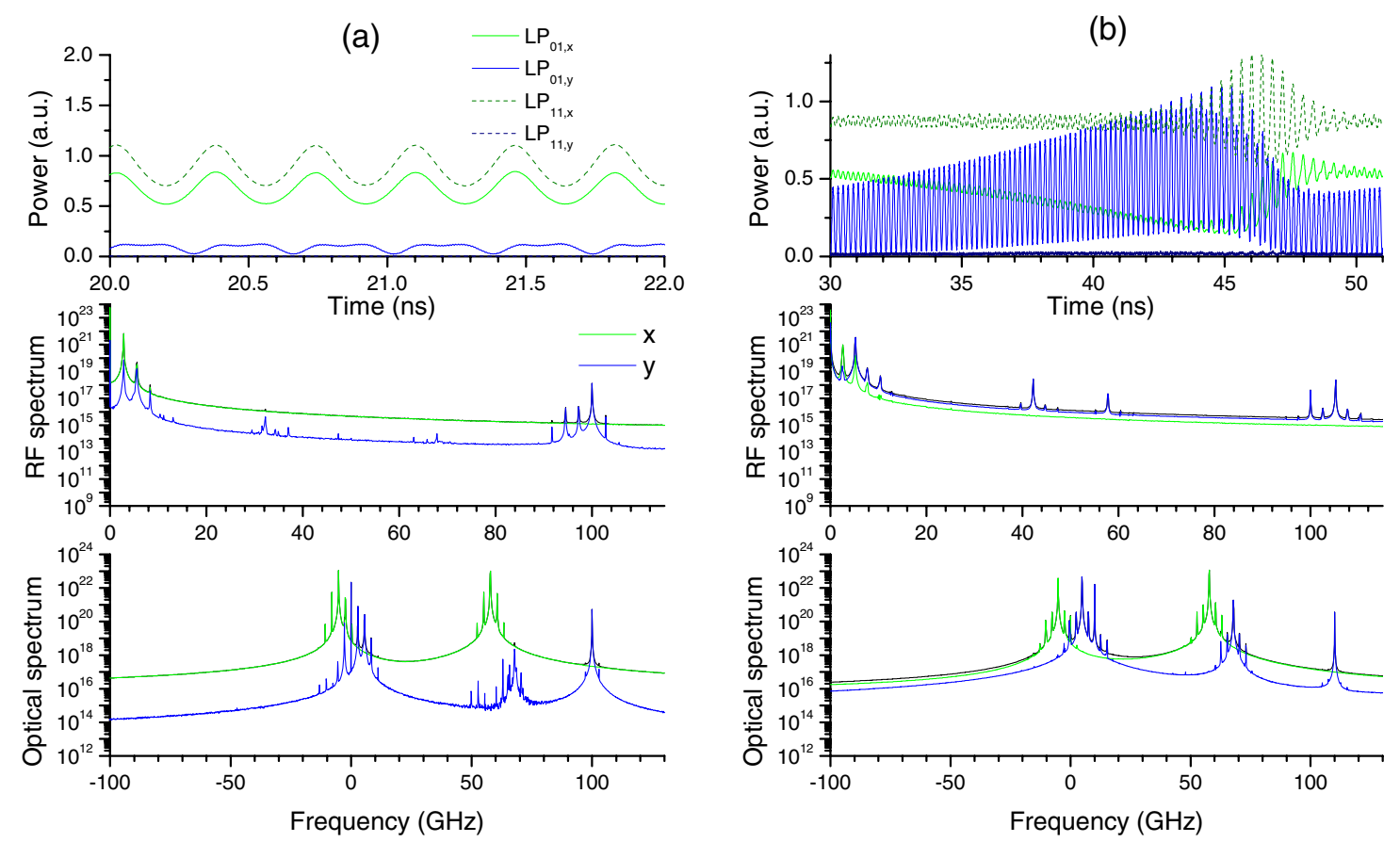

Fig. 9. (Color online) Temporal and spectral dynamics of the multi-transverse-mode VCSEL when $\Delta f=100 \mathrm{GHz}, \kappa_{s}=2 \cdot 10^{-4}$, and (a) $\Delta \nu=-5 \mathrm{GHz}$, (b) $\Delta \nu=5 \mathrm{GHz}$. Upper row: time traces of the power of the polarized transverse modes. Middle row: RF spectra of the polarized powers. Lower row: optical spectra of the $x$-polarized (grey curve), $y$-polarized (dark grey curve), and total power. 
envelope of $21 \mathrm{~ns}$ period (only one period is shown in the figure). Modal powers oscillate in-phase during the slow part of the envelope (from 30 to $43 \mathrm{~ns}$ ) with a $5.2 \mathrm{GHz}$ frequency. However, during the fast part of the envelope, from 43 to $50 \mathrm{~ns}$, modal powers oscillate with different frequencies: while $\mathrm{LP}_{01, x}$ and $\mathrm{LP}_{11, x}$ oscillate in-phase at $2.6 \mathrm{GHz}, \mathrm{LP}_{01, y}$ keeps on oscillating at $5.2 \mathrm{GHz}$. Future work will be devoted to an indepth analysis of the nonlinear dynamics of this system.

\section{SUMMARY AND CONCLUSIONS}

In this work we have made a theoretical study of the photonic microwave signal generation obtained when single- and multitransverse-mode VCSELs are subject to two-frequency orthogonal optical injection. Numerical simulations taking into account the extra degree of freedom given by the polarization of the devices show that broadly tunable microwave signals can be obtained in these systems. In that regime PS has occured, and hence the generated optical signals have the same linear polarization as the master lasers. For multi-transversemode VCSELs, the higher-order transverse mode is excited with a much larger amplitude than that of the fundamental transverse mode. The comparison with the case of a singletransverse-mode VCSEL subject to the same two-frequency orthogonal optical injection shows that multi-transverse-mode operation of the VCSEL enhances the performance of the microwave generation system. Periodic oscillations have been obtained for a very wide range of frequency detunings between the optical injections and transverse modes. Two relative maxima of the microwave signal amplitude have been obtained for two-frequency detunings between the master laser with the smallest frequency and the orthogonally polarized fundamental transverse mode of the VCSEL. Each relative maximum appears when the frequency of one of the master lasers is very close to the frequency of that fundamental transverse mode. Periodic oscillations have been demonstrated for symmetric and asymmetric values of the injection strengths. Wide tuning ranges, extending into the terahertz band, have been obtained in our system. Our results are qualitatively similar to those previously found for parallel optical injection. In this way microwave signals can be generated independently of the polarization of the master lasers.

\section{ACKNOWLEDGMENTS}

This work is supported by the Ministerio de Ciencia e Innovación under project TEC2009-14581-C02-02. A. Quirce was supported in part by the Fondo Social Europeo (FSE) under the programme Junta de Ampliación de Estudios (JAE-predoc). H. Lin acknowledges support from the National Science Foundation under Grant No. PHY-1068789.

\section{REFERENCES}

1. J. Ohtsubo, Semiconductor Lasers: Stability, Instability and Chaos, Springer Series in Optical Sciences (Springer, 2007).

2. F. Koyama, "Recent advances of VCSEL photonics," J. Lightwave Technol. 24, 4502-4513 (2006)

3. C. H. Chang, L. Chrostowski, and C. J. Chang-Hasnain, "Injection locking of VCSELs," IEEE J. Sel. Top. Quantum Electron. 9, 1386-1393 (2003)

4. D. Parekh, X. Zhao, W. Hofmann, M. C. Amann, L. A. Zenteno, and C. J. Chang-Hasnain, "Greatly enhanced modulation response of injection-locked multimode VCSELs," Opt. Express 16, 21582-21586 (2008).
5. H. Li, T. Lucas, J. G. McInerney, M. Wright, and R. A. Morgan, "Injection locking dynamics of vertical cavity semiconductor lasers under conventional and phase conjugate injection," IEEE J. Quantum Electron. 32, 227-235 (1996).

6. Z. G. Pan, S. Jiang, M. Dagenais, R. A. Morgan, K. Kojima, M. T. Asom, and R. E. Leibenguth, "Optical injection induced polarization bistability in vertical-cavity surface-emitting lasers," Appl. Phys. Lett. 63, 2999-3001 (1993).

7. Y. Hong, K. A. Shore, A. Larsson, M. Ghisoni, and J. Halonen, "Pure frequency-polarisation bistability in vertical-cavity surface-emitting lasers subject to optical injection," Electron. Lett. 36, 2019-2020 (2000).

8. J. Buesa, I. Gatare, K. Panajotov, H. Thienpont, and M. Sciamanna, "Mapping of the dynamics induced by orthogonal optical injection in vertical-cavity surface-emitting lasers," IEEE J. Quantum Electron. 42, 198-207 (2006).

9. K. Panajotov, I. Gatare, A. Valle, H. Thienpont, and M. Sciamanna, "Polarization- and transverse-mode dynamics in optically injected and gain-switched vertical-cavity surfaceemitting lasers," IEEE J. Quantum Electron. 45, 1473-1481 (2009).

10. A. Valle, M. Gomez-Molina, and L. Pesquera, "Polarization bistability in $1550 \mathrm{~nm}$ wavelength single-mode vertical-cavity surface-emitting lasers subject to orthogonal optical injection," IEEE J. Sel. Top. Quantum Electron. 14, 895-902 (2008).

11. A. Hurtado, I. D. Henning, and M. J. Adams, "Two-wavelength switching with a $1550 \mathrm{~nm}$ VCSEL under single orthogonal optical injection,” IEEE J. Sel. Top. Quantum Electron. 14, 911-917 (2008).

12. K. H. Jeong, K. H. Kim, S. H. Lee, M. H. Lee, B. S. Yoo, and K. A. Shore, "Optical injection-induced polarization switching dynamics in $1.5 \mu \mathrm{m}$ wavelength single-mode vertical-cavity surfaceemitting lasers," IEEE Photon. Technol. Lett. 20, 779-781 (2008).

13. A. Valle, I. Gatare, K. Panajotov, and M. Sciamanna, "Transverse mode switching and locking in vertical-cavity surface-emitting lasers subject to orthogonal optical injection,” IEEE J. Quantum Electron. 43, 322-333 (2007)

14. A. Quirce, J. R. Cuesta, A. Valle, A. Hurtado, L. Pesquera, and M. J. Adams, "Polarization bistability induced by orthogonal optical injection in $1550 \mathrm{~nm}$ multimode VCSELs," IEEE J. Sel. Top. Quantum Electron. 18, 772-778 (2012).

15. H. Lin, Y. Zhang, D. W. Pierce, A. Quirce, and A. Valle, "Polarization dynamics of a multimode vertical-cavity surface-emitting laser subject to orthogonal optical injection,” J. Opt. Soc. Am. B 29, 867-873 (2012).

16. C. J. Chang-Hasnain, J. P. Harbison, G. Hasnain, A. C Von Lehmen, L. T. Florez, and N. G. Stoffel, "Dynamic, polarization and transverse mode characteristics of vertical-cavity surface-emitting lasers," IEEE J. Quantum Electron. 27, 1402-1409 (1991).

17. A. Valle, J. Sarma, and K. A. Shore, "Spatial holeburning effects on the dynamics of vertical cavity surface-emitting laser diodes," IEEE J. Quantum Electron. 31, 1423-1431 (1995).

18. K. D. Choquette, R. P. Schneider, K. L. Lear, and R. E. Leibenguth "Gain-dependent polarization properties of vertical-cavity lasers," IEEE J. Sel. Top. Quantum Electron. 1, 661-666 (1995).

19. A. Hayat, A. Bacou, A. Rissons, J. C. Mollier, V. Iakovlev, A. Sirbu, and E. Kapon, "Long-wavelength VCSEL-by-VCSEL optical injection locking," IEEE Trans. Microwave Theory Tech. 57, 1850-1858 (2009).

20. H. Lin, D. W. Pierce, A. J. Basnet, A. Quirce, Y. Zhang, and A. Valle, "Two-frequency injection on a multimode vertical-cavity surface-emitting laser," Opt. Express 19, 22437-22442 (2011).

21. S. C. Chan, R. Diaz, and J. M. Liu, "Novel photonic applications of nonlinear semiconductor laser dynamics," Opt. Quantum Electron. 40, 83-95 (2008).

22. S. C. Chan, S. K. Hwang, and J. M. Liu, "Radio-over fiber transmission from an optically injected semiconductor laser in period-one state," Opt. Express 15, 14921-14935 (2007).

23. X. Q. Qi and J. M. Liu, "Photonic microwave applications of the dynamics of semiconductor lasers," IEEE J. Sel. Top. Quantum Electron. 17, 1198-1211 (2011).

24. S. C. Chan, "Analysis of an optically injected semiconductor laser for microwave generation," IEEE J. Quantum Electron. 46, 421-428 (2010). 
25. S. C. Chan, S. K. Hwang, and J. M. Liu, "Radio-over-fiber AMto-FM upconversion using an optically injected semiconductor laser," Opt. Lett. 31, 2254-2256 (2006).

26. C. Cui and S. C. Chan, "Performance analysis on using periodone oscillation of optically injected semiconductor lasers for radio-over-fiber uplinks," IEEE J. Quantum Electron. 48, 490-499 (2012).

27. X. Q. Qi and J. M. Liu, "Dynamic scenarios of dual-beam optically injected semiconductor lasers," IEEE J. Quantum Electron. 47, 762-769 (2011).

28. Y. S. Juan and F. Y. Lin, "Photonic generation of broadly tunable microwave signals utilizing a dual-beam optically injected semiconductor laser," IEEE Photon. J. 3, 644-650 (2011).

29. Y. C. Chen, Y. S. Juan, and F. Y. Lin, "High-frequency microwave signal generation in a semiconductor laser under double injection locking," Proc. SPIE 7936, 793609 (2011).

30. A. Quirce and A. Valle, "High-frequency microwave signal generation using multi-transverse mode VCSELs subject to two-frequency optical injection," Opt. Express 20, 13390-13401 (2012).

31. A. Valle, K. A. Shore, and L. Pesquera, "Polarization selection in birefringent vertical cavity surface emitting lasers," J. Lightwave Technol. 14, 2062-2068 (1996).
32. G. H. M. Van Tartwijk and D. Lenstra, "Semiconductor lasers with optical injection and feedback," Quantum Semiclass. Opt. 7, 87-143 (1995).

33. I. Gatare, M. Sciamanna, M. Nizette, and K. Panajotov, "Bifurcation to polarization switching and locking in vertical-cavity surface-emitting lasers with optical injection," Phys. Rev. A 76, 031803(R) (2007).

34. J. Dellunde, A. Valle, L. Pesquera, and K. A. Shore, "Transversemode selection and noise properties of external-cavity surfaceemitting lasers including multiple reflection effects," J. Opt. Soc. Am. B 16, 2131-2139 (1999).

35. A. Valle and L. Pesquera, "Relative intensity noise of multitransverse-mode vertical-cavity surface-emitting lasers," IEEE Photon. Technol. Lett. 13, 272-274 (2001).

36. A. Valle and L. Pesquera, "Theoretical calculation of relative intensity noise of multimode vertical-cavity surfaceemitting lasers," IEEE J. Quantum Electron. 40, 597-606 (2004).

37. J. Javaloyes and S. Balle, "Quasiequilibrium time-domain susceptibility of semiconductor quantum wells," Phys. Rev. A 81 062505 (2010).

38. A. Valle, H. Lin, Z. J. Lapin, and B. Malla, "Analysis of the polarization dynamics in a multitransverse-mode vertical-cavity surface-emitting laser with isotropic optical feedback," Phys. Rev. A 78, 033828 (2008). 